\title{
Effective slip for flow through a channel bounded by lubricant-impregnated grooved surfaces
}

\author{
Rui Sun · Chiu-On Ng
}

Received: date / Accepted: date

\begin{abstract}
This study aims to investigate effective slip arising from pressure-driven flow through a slit channel bounded by lubricant-impregnated grooved surfaces. The problem for flow over longitudinal grooves is solved analytically using the methods of domain decomposition and eigenfunction expansion, while that for flow over transverse grooves is solved numerically using the front tracking method. It is found that the effective slip length and the lubricant flow rate can depend strongly on the geometry of the microstructure, the direction of flow, as well as the lubricant viscosity. In particular, the effective slip can be effectively enhanced by increasing the thickness of a lubricating film atop the ribs. Under the same conditions, a flow that is parallel to the lubricant-impregnated grooves will have a larger effective slip, but also a larger lubricant flow rate, when compared with the case of flow normal to the grooves. It is also shown that, in the case of transverse grooves, because of the downward displacement of the interface between the working/lubricating fluids, the effective slip length and lubricant flow rate may vary non-monotonically with the groove depth.
\end{abstract}

Keywords Lubricant impregnated surface · effective slip · eigenfunction expansion · front tracking method

Rui Sun

Department of Mechanical Engineering

The University of Hong Kong

Pokfulam Road, Hong Kong

Chiu-On Ng

Department of Mechanical Engineering

The University of Hong Kong

Pokfulam Road, Hong Kong

Tel.: +852-28592622

Fax: +852-2858 5415

E-mail: cong@hku.hk 


\section{Introduction}

Slip arising from superhydrophobicity over a microstructured surface has long been a subject of interest in interfacial science [21]. Recent advances have enabled the realization of microstructured surfaces that can further achieve omniphobicity. Inspired by Nepenthes pitcher plants [2], the surfaces have been known by various names, such as slippery liquid-infused porous surface (SLIPS) [35], slippery presuffused surface [8], or lubricant impregnated surface (LIS) [1]. In essence, these kinds of surfaces are created by infusing a lubricating liquid into a functionalized microtextured substrate. Experiments by Wong et al. [35] showed that SLIPS can be in many aspects superior to a superhydrophobic surface on functioning as a slippery surface. While a superhydrophobic surface provides effective slip via an air-liquid interface, a SLIPS works by virtue of a liquid-liquid interface. In this regard, SLIPS can repel a broader variety of liquids, such as water and hydrocarbons (i.e., it is both hydrophobic and oleophobic, thereby omniphobic). In recent years, SLIPS or LIS have been intensively studied, exploring its various capabilities: a low contact angle hysteresis, self-cleaning, quick self-repairing after physical damage, ice repellency, dropwise condensation or fog harvesting, stability under high pressure, anti-fouling, enhanced optical transparency, and so on. See, among many others, Refs. [3-7, 9$11,18,23,24,28,30,34,36,37]$ for some details about these potential capabilities of SLIPS. Above all, it is durable, robust and inexpensive. All these characteristics have made SLIPS or LIS a very promising material surface for many applications where liquid/ice repellency or anti-fouling is wanted.

The laboratory study by Solomon et al. [29] has in particular shown that LIS may generate significant drag reduction in viscous laminar flow. For a sufficiently low lubricant to working fluid viscosity ratio, a remarkable drag reduction of $16 \%$, corresponding to an effective slip length of 18 microns, could be achieved. Also, the numerical study by Rajagopal et al. [22] showed that a drag reduction as much as $14 \%$ could be obtained using a viscoelastic liquid infused microtextured surface. These experimental and numerical evidences, while preliminary, suggest that LIS can be an excellent alternative to superhydrophobic surfaces, which are often used to reduce drag for flow through a microchannel.

In terms of applications, SLIPS or LIS is still in a very early stage of development. Much about its performance remains to be understood. Thus far, most studies have been focused on the mobility of droplets on the surface. There virtually exist no studies investigating in detail the hydrodynamics occurring within and outside the surface texture under various flow conditions. Wong et al. [35] have remarked that the liquid repellency of SLIPS is insensitive to the geometry of the underlying substrate, as long as the texture is covered by a lubricating film. This is, however, not necessarily true when SLIPS is used to achieve effective boundary slip in a pressure-driven internal flow. For such flow, the effective slip will, on the contrary, strongly depend on the geometry (e.g., two-dimensional ribs versus three-dimensional posts) of the microtexture, whether or not covered by a lubricating film. This is a core issue that needs to be addressed in order to quantify the effective slip or drag reduction that can be derived from a SLIPS or LIS. 
The present study aims to investigate effective slip that can be accomplished using a lubricant impregnated surface in pressure-driven flow through a slit channel. For simplicity of modeling, a channel wall that is patterned with a two-dimensional periodic array of lubricant-impregnated grooves is considered. This will enable us to investigate the two limiting cases, where the flow can be in a direction parallel or normal to the wall pattern, corresponding to the upper and lower bounds of effective slip that can be achieved with the same surface under a fixed set of flow conditions.

In addition to effective slip, we shall also look into the discharge of the lubricant in the course of lubricating the flow of the working fluid. This drainage or discharge of lubricant over time is one of the concerns in the use of LIS [29,33].

Schönecker and Hardt [26, 27] and Schönecker et al. [25] have recently studied longitudinal and transverse flow over a single cavity or a periodic array of rectangular cavities containing a second immiscible fluid. In their models, flow in the cavity is approximated into a Navier slip boundary condition to the outer flow, where the locally varying slip length is assumed to have an elliptic or a corresponding distribution, the peak of which can be determined heuristically from the related problems of lid-driven flow. They have found good agreement between results generated by their models and those by solving the Navier-Stokes equations numerically. Their models are, however, limited in the following aspects. First, a flat (i.e., undeformed) interface between the outer and cavity fluids is assumed. Second, the interface is assumed to be located exactly at the top of the cavity. Third, a semi-infinite domain of outer fluid is assumed. Applying the assumption of a flat interface to flow over a transverse cavity is subject to scrutiny, since the flow field will generate a pressure that is nonuniformly distributed along the interface, which will then be deformed following the flow dynamics. The interfacial deformation over a cavity can be large if the cavity is sufficiently wide. Also, in practice, there is a tendency for the interface to be positioned slightly above the top of the cavity, forming a thin lubricating film above the structured surface. In the present study, we shall look into problems incorporating these effects: a deformable interface between the working and lubricating fluids in the case of transverse flow, and a thin lubricating film above the structured surface in the case of longitudinal flow. We shall also look into effect due to a finite channel height, or wall confinement.

Our problems for flow over longitudinal and transverse grooves are described in further detail in Secs. 2 and 3, respectively. We shall determine for each problem the effective slip and lubricant flow rate as functions of geometrical parameters (such as the width and depth of the grooves, thickness of the working fluid layer, and thickness of the lubricant film atop the ribs), and fluid properties (such as the viscosity of the lubricant). The problem for longitudinal grooves, which is one dimensional, can be solved semi-analytically using the methods of domain decomposition and eigenfunction expansion. The problem for transverse grooves, which is two dimensional involving an undetermined interface separating the working and lubricating fluids, can only be solved numerically. We have chosen to use the front tracking numerical method to solve this problem of two-phase flow with a movable interface. We shall present numerical results to reveal how the controlling parameters may have similar or dissimilar effects on the effective slip and lubricant flow rate, depending on whether the flow is parallel or normal to the grooves. 


\section{Longitudinal grooves}

\subsection{Problem formulation}

As stated above, the present study aims to look into pressure-driven flow through a slit channel bounded by two identical walls which are each patterned with a twodimensional periodic array of rectangular grooves infused with a lubricating fluid. In this section, flow that is parallel to the grooves, which are then referred to as longitudinal grooves, is considered. We shall, for simplicity of analysis, assume that the two wall patterns are aligned in phase, and therefore by symmetry, it suffices for us to consider flow in one half of the channel. We show in Fig. 1 a definition sketch of the problem, where the flow is perpendicular to the plane of paper. Unless stated otherwise, we shall express equations and solutions in terms of non-dimensional quantities. The lengths are normalized by $L$, and the velocities are normalized by $K_{z} L^{2} / \mu_{w}$, where $L$ is half the wall pattern period, $K_{z}=-\partial p / \partial z$ is the axial pressure gradient applied to the fluids, and $\mu_{w}$ is the dynamic viscosity of the working fluid. The ratio of the viscosity of the lubricating fluid $\left(\mu_{o}\right)$ to that of the working fluid $\left(\mu_{w}\right)$ is denoted by $\eta=\mu_{o} / \mu_{w}$.

To allow analytical analysis, the interfacial curvature due to differential wetting of the solid by the two fluids is ignored. In other words, a special contact angle of the fluids with the wall of $90^{\circ}$ is assumed. As a result, the Laplace pressure difference across the interface is equal to zero. We further assume that the pressure varies only linearly in the $z$-direction (due to the externally applied pressure forcing), and is invariant in the spanwise and vertical ( $x$ and $y$ ) directions. Locally the pressure is uniform across the channel section. In this regard, the flow is strictly unidirectional along the $z$-direction. The flow kinematics and dynamics therefore allow us to consider a flat interface between the two fluids in the case of longitudinal flow. The problem here is to solve the $z$-momentum equation for the axial velocity $w(x, y)$. Non-wetting effect due to chemical coating of solid surfaces or intrinsic slippage is also ignored.

Two possible configurations are shown in Fig. 1: the interface between the two fluids can be located above or below the top of the ribs and grooves. Our primary focus is on the first case, shown in Fig. 1(a), which is normally expected of a lubricantimpregnated surface. In this configuration, the lubricating fluid not only completely fills the grooves, but also covers the ribs with a thin film. Here, the interface between the working and lubricating fluids is located at a height $h_{0}$ above the top of the ribs and grooves, and each groove has a width of $2 a$ and depth $b$. In this flow configuration, the working fluid, which is confined to a bulk region of height $2 h$, is completely lubricated as it does not have direct contact with the solid walls. We decompose the domain of analysis, which covers one half of a wall unit $(0 \leq x \leq 1)$, into three regions, as shown in Fig. 1(a). For convenience, we develop solutions for the flow of the working fluid in Region I, and that of the lubricating fluid in Regions II and III in terms of the wall-normal coordinates $y$ and $y^{\prime}$, respectively. Flow is purely one dimensional along the $z$-direction, where the velocity $w(x, y)$ may be found using the methods of domain decomposition and eigenfunction expansion [13-15] as described below. By symmetry, the velocity gradient $\partial w / \partial x$ is zero at $x=0$ and $x=1$. 
(a) Interface above top of ribs $\left(h_{0}>0\right)$

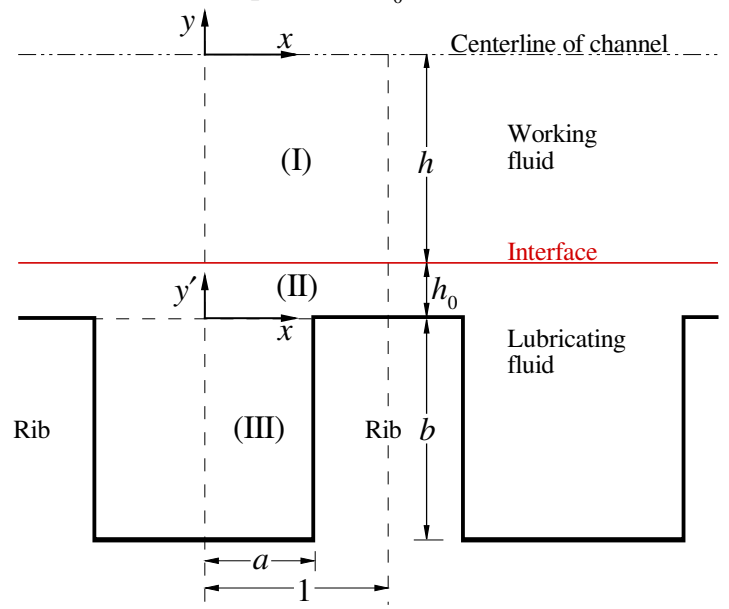

(b) Interface below top of ribs $\left(h_{0}<0\right)$

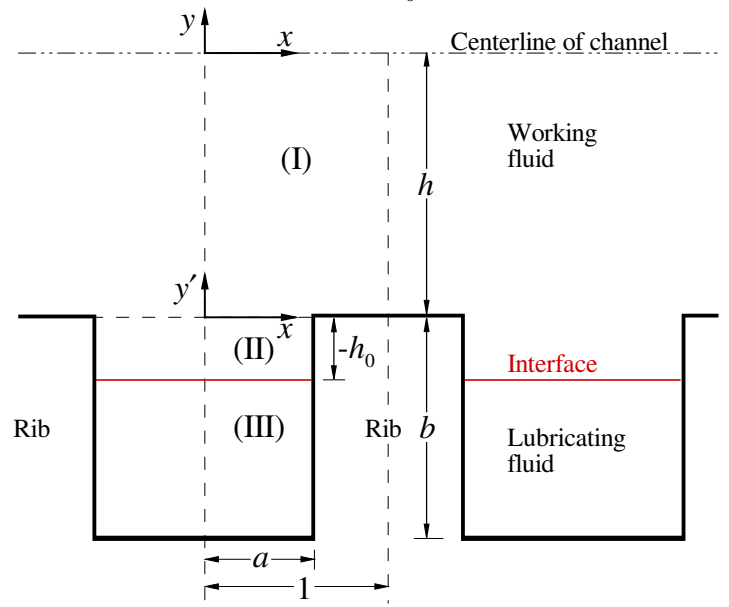

Fig. 1 Definition sketch of flow over longitudinal grooves, where the flow is driven by a pressure gradient perpendicular to the plane of paper, and (a) the interface is above the top of the ribs, or (b) the interface is below the top of the ribs.

When the lubricating fluid dwindles in volume as a result of discharge, the interface between the two fluids may fall below the top of the ribs, as is shown in Fig. 1(b). In this configuration, the domain can be decomposed into Regions I and II, which are occupied by the working fluid, and Region III, which is occupied by the lubricating fluid. Likewise, the problem can be solved using the methods of domain decomposition and eigenfunction expansion. We shall present results, but not the mathematical details, for this case. 


\subsection{Semi-analytical solution}

Let us consider the flow depicted in Fig. 1(a). In Region I $(-h \leq y \leq 0)$, which is occupied by the working fluid, the governing equation is

$$
\frac{\partial^{2} w_{\mathrm{I}}}{\partial x^{2}}+\frac{\partial^{2} w_{\mathrm{I}}}{\partial y^{2}}=-1
$$

The solution satisfying symmetry about $y=0$ is given by

$$
w_{\mathrm{I}}(x, y)=\frac{1}{2}\left(h^{2}-y^{2}\right)+A_{0}+\sum_{n=1}^{\infty} A_{n} \cos \left(\alpha_{n} x\right) \frac{\cosh \left(\alpha_{n} y\right)}{\cosh \left(\alpha_{n} h\right)},
$$

where $A_{0,1, \ldots}$ are undetermined coefficients and

$$
\alpha_{n}=n \pi
$$

In Region II $\left(0 \leq y^{\prime} \leq h_{0}\right)$ and Region III $\left(-a \leq x \leq a,-b \leq y^{\prime} \leq 0\right)$, which are occupied by the lubricating fluid, the governing equation is the same:

$$
\frac{\partial^{2} w_{\mathrm{IIIIII}}}{\partial x^{2}}+\frac{\partial^{2} w_{\mathrm{II}, \mathrm{III}}}{\partial y^{2}}=-\frac{1}{\eta} .
$$

The solution for Region II is expressible by

$$
w_{\text {II }}(x, y)=-\frac{y^{\prime 2}}{2 \eta}+B_{0} y^{\prime}+C_{0}+\sum_{n=1}^{\infty} \cos \left(\alpha_{n} x\right)\left[B_{n} \sinh \left(\alpha_{n} y^{\prime}\right)+C_{n} \cosh \left(\alpha_{n} y^{\prime}\right)\right],
$$

while that for Region III satisfying no-slip at $y^{\prime}=-b$ can be written as

$$
\begin{aligned}
w_{\text {III }}(x, y)= & -\frac{1}{2 \eta}\left(y^{\prime 2}+b y^{\prime}\right)+\sum_{n=1}^{\infty} D_{n} \cos \left(\beta_{n} x\right)\left[e^{\beta_{n} y^{\prime}}-e^{-\beta_{n}\left(y^{\prime}+2 b\right)}\right] \\
& +\sum_{n=1}^{\infty} E_{n} \sin \left(\gamma_{n} y^{\prime}\right) \frac{\cosh \left(\gamma_{n} x\right)}{\cosh \left(\gamma_{n} a\right)},
\end{aligned}
$$

where $B_{0,1, \ldots}, C_{0,1, \ldots,}, D_{1, \ldots}$ and $E_{1, \ldots}$ are undetermined coefficients, and

$$
\beta_{n}=(n-1 / 2) \pi / a, \quad \gamma_{n}=n \pi / b .
$$

The coefficients are to be determined using the matching and boundary conditions, as detailed below. In this regard, we need to truncate the series to finite terms. Let us truncate coefficients $A_{n}, B_{n}, C_{n}$ each to $M$ terms, $D_{n}$ to $N$ terms, and $E_{n}$ to $P$ terms, where $N \approx \operatorname{Integer}(a M)$ and $P \approx \operatorname{Integer}(b M)$. We are going to develop linear equations to be solved for these coefficients. According to previous studies [14, 32], $M \sim 100$ is sufficient to generate accurate results.

The continuity of velocity and shear stress on the interface, $w_{\mathrm{I}}(y=-h)=w_{\mathrm{II}}\left(y^{\prime}=\right.$ $\left.h_{0}\right)$ and $\partial w_{\mathrm{I}} /\left.\partial y\right|_{y=-h}=\eta \partial w_{\mathrm{II}} /\left.\partial y^{\prime}\right|_{y^{\prime}=h_{0}}$, give

$$
\begin{aligned}
A_{0} & +\sum_{n=1}^{M} A_{n} \cos \left(\alpha_{n} x\right) \\
= & -\frac{h_{0}^{2}}{2 \eta}+h_{0} B_{0}+C_{0}+\sum_{n=1}^{M}\left[B_{n} \sinh \left(\alpha_{n} h_{0}\right)+C_{n} \cosh \left(\alpha_{n} h_{0}\right)\right] \cos \left(\alpha_{n} x\right),
\end{aligned}
$$




$$
\begin{aligned}
h & -\sum_{n=1}^{M} \alpha_{n} A_{n} \tanh \left(\alpha_{n} h\right) \cos \left(\alpha_{n} x\right) \\
& =-h_{0}+\eta B_{0}+\eta \sum_{n=1}^{M} \alpha_{n}\left[B_{n} \cosh \left(\alpha_{n} h_{0}\right)+C_{n} \sinh \left(\alpha_{n} h_{0}\right)\right] \cos \left(\alpha_{n} x\right) .
\end{aligned}
$$

The continuity of velocity on the top of the groove together with the no-slip condition on the top of the rib:

$$
w_{\mathrm{II}}=\left\{\begin{array}{ll}
w_{\mathrm{III}} & 0 \leq x<a, \\
0 & a<x \leq 1,
\end{array} y^{\prime}=0\right.
$$

gives

$$
C_{0}+\sum_{n=1}^{M} C_{n} \cos \left(\alpha_{n} x\right)=\left\{\begin{array}{ll}
\sum_{n=1}^{N} D_{n}\left(1-e^{-2 \beta_{n} b}\right) \cos \left(\beta_{n} x\right) & 0 \leq x<a, \\
0 & a<x \leq 1,
\end{array} .\right.
$$

The continuity of shear stress on the top of the groove:

$$
\frac{\partial w_{\mathrm{II}}}{\partial y^{\prime}}=\frac{\partial w_{\mathrm{III}}}{\partial y^{\prime}} \quad 0 \leq x \leq a, y^{\prime}=0
$$

gives

$$
\begin{aligned}
B_{0} & +\sum_{n=1}^{M} \alpha_{n} B_{n} \cos \left(\alpha_{n} x\right) \\
= & -\frac{b}{2 \eta}+\sum_{n=1}^{N} \beta_{n} D_{n}\left(1+e^{-2 \beta_{n} b}\right) \cos \left(\beta_{n} x\right)+\sum_{n=1}^{P} \gamma_{n} E_{n} \frac{\cosh \left(\gamma_{n} x\right)}{\cosh \left(\gamma_{n} a\right)} .
\end{aligned}
$$

Finally, the no-slip condition on the lateral wall of the groove, $w_{\mathrm{III}}\left(x=a,-b \leq y^{\prime} \leq\right.$ $0)=0$, gives

$$
\sum_{n=1}^{P} E_{n} \sin \left(\gamma_{n} y^{\prime}\right)=\frac{1}{2 \eta}\left(y^{\prime 2}+b y^{\prime}\right) .
$$

On matching terms, we get from Eqs. (8) and (9)

$$
\begin{gathered}
A_{0}=-\frac{h_{0}^{2}}{2 \eta}+h_{0} B_{0}+C_{0}, \\
B_{0}=\frac{1}{\eta}\left(h+h_{0}\right), \\
A_{n}=\frac{\eta \operatorname{sech}\left(\alpha_{n} h_{0}\right) C_{n}}{\tanh \left(\alpha_{n} h\right) \tanh \left(\alpha_{n} h_{0}\right)+\eta}, \\
B_{n}=-\frac{\tanh \left(\alpha_{n} h\right)+\eta \tanh \left(\alpha_{n} h_{0}\right)}{\tanh \left(\alpha_{n} h\right) \tanh \left(\alpha_{n} h_{0}\right)+\eta} C_{n} .
\end{gathered}
$$

On integrating Eq. (11) with respect to $x$ from 0 to 1 , we get

$$
C_{0}=\sum_{n}^{N} D_{n} \frac{(-1)^{n+1}}{\beta_{n}}\left(1-e^{-2 \beta_{n} b}\right)
$$


For $m=1, \cdots, M$, we multiply Eq. (11) by $\cos \left(\alpha_{m} x\right)$, which is then integrated with respect to $x$ from 1 to 0 . This gives

$$
\frac{1}{2} C_{m}-\sum_{n=1}^{N} I_{m n}\left(1-e^{-2 \beta_{n} b}\right) D_{n}=0,
$$

where

$$
\begin{aligned}
I_{m n} & =\int_{0}^{a} \cos \left(\alpha_{m} x\right) \cos \left(\beta_{n} x\right) \mathrm{d} x \\
& = \begin{cases}\frac{\sin \left[\left(\alpha_{m}-\beta_{n}\right) a\right]}{2\left(\alpha_{m}-\beta_{n}\right)}+\frac{\sin \left[\left(\alpha_{m}+\beta_{n}\right) a\right]}{2\left(\alpha_{m}+\beta_{n}\right)} & \text { for } \alpha_{m} \neq \beta_{n} \\
\frac{a}{2} & \text { for } \alpha_{m}=\beta_{n}\end{cases}
\end{aligned}
$$

For $m=1, \cdots, P$, we multiply Eq. (14) by $\sin \left(\gamma_{m} y^{\prime}\right)$, which is then integrated with respect to $y^{\prime}$ from $-b$ to 0 . This gives

$$
E_{m}=\frac{2}{\eta \gamma_{m}^{3} b}\left[1+(-1)^{m+1}\right] .
$$

For $m=1, \cdots, N$, we multiply Eq. (13) by $\cos \left(\beta_{m} x\right)$, which is then integrated with respect to $x$ from 0 to $a$. This gives, after substituting Eqs. (18) and (22),

$$
\begin{aligned}
& \sum_{n=1}^{M} \alpha_{n} I_{n m} \frac{\left[\tanh \left(\alpha_{n} h\right)+\eta \tanh \left(\alpha_{n} h_{0}\right)\right]}{\left[\tanh \left(\alpha_{n} h\right) \tanh \left(\alpha_{n} h_{0}\right)+\eta\right]} C_{n}+\frac{\beta_{m} a}{2}\left[1+e^{-2 \beta_{m} b}\right] D_{m} \\
& =\left(\frac{b}{2 \eta}+B_{0}\right) \frac{(-1)^{m+1}}{\beta_{m}}-\sum_{n=1}^{P} \frac{2 \beta_{m}(-1)^{m+1}\left[1+(-1)^{n+1}\right]}{\eta \gamma_{n}^{2} b\left(\beta_{m}^{2}+\gamma_{n}^{2}\right)} .
\end{aligned}
$$

Equations (20) and (23) constitute a system of $M+N$ linear equations that can be solved, using a standard routine, for the coefficients $C_{1, \cdots, M}$ and $D_{1, \cdots, N}$. The other coefficients may then be determined from Eqs. (15), (17)-(19).

The volume flow rate of the working fluid per unit width of the channel is

$$
q=2 \int_{-h}^{0} \int_{0}^{1} w_{\mathrm{I}} \mathrm{d} x \mathrm{~d} y=\frac{2}{3} h^{3}+2 h A_{0} .
$$

By equating this flow rate to that through a slit channel of height $2 h$ bounded by plane walls of slip length $\lambda: q=2 h^{3} / 3+2 h^{2} \lambda$, we may deduce that the effective slip length for the longitudinal grooves is given by

$$
\lambda_{\|}=\frac{A_{0}}{h}=\frac{1}{2 \eta}\left(2 h_{0}+\frac{h_{0}^{2}}{h}\right)+\frac{C_{0}}{h} .
$$

Note that the effective slip length deduced this way is a macroscopic or system specific quantity; it is the boundary slip length of an equivalent uniform channel of the same height giving rise to the same rate of flow of the working fluid under the same pressure forcing. A positive effective slip length amounts to flow enhancement, which has a clear meaning here. It refers to how much the flow rate is increased when compared with the base case of flow through the same equivalent uniform channel but without boundary slip. 
The flow rate of the lubricating fluid per unit width on one side of the channel is

$$
\begin{aligned}
q_{o}= & \int_{0}^{h_{0}} \int_{0}^{1} w_{\mathrm{II}} \mathrm{d} x \mathrm{~d} y^{\prime}+\int_{-b}^{0} \int_{0}^{a} w_{\mathrm{II}} \mathrm{d} x \mathrm{~d} y^{\prime} \\
= & \frac{h_{0}^{2}}{\eta}\left(\frac{h}{2}+\frac{h_{0}}{3}\right)+h_{0} C_{0}+\frac{a b^{3}}{12 \eta}+\sum_{n=1}^{N} \frac{D_{n}(-1)^{n+1}}{\beta_{n}^{2}}\left(1-e^{-\beta_{n} b}\right)^{2} \\
& -\sum_{n=1}^{P} \frac{2 \tanh \left(\gamma_{n} a\right)}{\eta \gamma_{n}^{5} b}\left[1+(-1)^{n+1}\right]^{2} .
\end{aligned}
$$

The solution given above is valid even for the limiting case $h_{0}=0$ (i.e., the vanishing of Region II). It is also of interest to consider $h_{0}<0$, corresponding to the case where the interface between the working/lubricating fluids falls below the top of the ribs, and the lubricant is completely inside the grooves; see Fig. 1(b). In this case the lubricating film above the ribs disappears, thereby causing the working fluid to be in contact with the top portion of the ribs, possibly resulting in a smaller lubricating effect compared with the case shown in Fig. 1(a) if the lubricant is less viscous than the working fluid $(\eta<1)$. The solution for the problem with a negative $h_{0}$ can be deduced in a manner similar to that presented above. The effective slip length is also derived in a similar manner, i.e., by equating the flow rate of the working fluid to that through a slit channel of uniform height $2 h$ and constant wall slip length $\lambda$. In the interest of space, the details of the solution are omitted here, but the case of $h_{0}<0$ will be included in our numerical discussion below.

\subsection{Results and discussion}

To demonstrate accuracy of our model, let us first compare results with those by previous studies. We show in Fig. 2 the longitudinal slip length $\lambda_{\|}$as a function of the groove area fraction $a$, for the limiting case $h \gg 1$ (very thick channel) and $h_{0}=0$ (interface at the top of the grooves), and a unity aspect ratio of the grooves $b=2 a$. For $\eta>0$, our results are found to agree very well with those presented in Fig. 7(a) of Schönecker et al. [25], despite a different approach of modeling used by these authors. When the lubricating fluid becomes inviscid $\eta=0$, the problem reduces to the classical problem studied by Philip [20] for Stokes flow over a surface with longitudinal alternating no-shear and no-slip slots. The formula to calculate the effective slip length for this classical problem is well known:

$$
\lim _{\substack{h \gg 1 \\ h=0 \\ \eta=0}} \lambda_{\|}=\frac{2}{\pi} \ln \left[\sec \left(\frac{\pi a}{2}\right)\right] .
$$

Again, for $\eta=0$, our results are found in close agreement with those computed by the formula above. Using $M=100$, we have obtained results which differ from the analytical values by a relative difference of $10^{-3}$ or smaller.

We next show in Fig. 3 the slip length $\lambda_{\|}$, and the lubricant flow rate $q_{o}$, as functions of the height of the interface above the top of the ribs (i.e., the lubricant film 


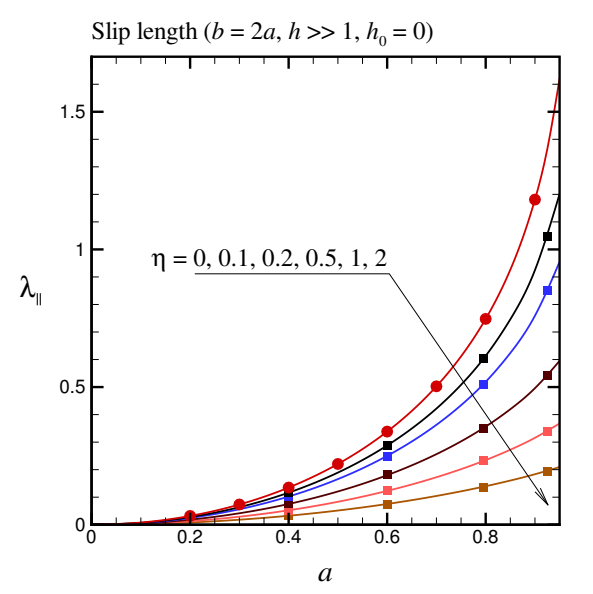

Fig. 2 For longitudinal grooves, the effective slip length $\lambda_{\|}$as a function of the groove area fraction $a$ and the viscosity ratio $\eta$, where $b=2 a, h \gg 1$ and $h_{0}=0$. The squares (for $\eta>0$ ) are from Schönecker et al. [25], and the circles (for $\eta=0$ ) are from Eq. (27).

thickness) $h_{0}$, and the groove area fraction $0<a \leq 1$. The limiting case of $a=1$ corresponds to thin fins. When $h_{0}<0$ (i.e., lubricant completely inside the grooves), the slip length increases only mildly as the interface increases in height (more lubricant filling the grooves). In sharp contrast, when $h_{0}>0$ (i.e., the interface lies atop the grooves and ribs), the slip length increases much more rapidly as $h_{0}$ increases. For small $\eta$, this effect becomes more pronounced for larger $a$. It is remarkable that at $a=1, \lambda_{\|}$can increase dramatically as $h_{0}$ turns slightly from negative to positive; see Fig. 3(c). Our results thereby confirm that the lubricant film atop the ribs indeed plays an important role in lubricating the working fluid flow. For a sufficiently thick lubricant film, the effective slip length increases in a nearly linear manner with the film thickness, where the rate of increase is larger for a smaller viscosity ratio $\eta$.

A larger effective slip is, however, accompanied by a larger flow rate of the lubricating fluid. Figures 3(b,d) show that $q_{o}$ has largely similar dependence on the parameters $a$ and $h_{0}$ as $\lambda_{\|}$does. A less viscous lubricant gives rise to a higher slip length, but also gives rise to a larger flow rate of the lubricant itself. This is because, in this longitudinal configuration, the lubricant, although mostly stored in the grooves, is driven by the same pressure gradient as the working fluid is. We therefore conclude that, for longitudinal grooves, a thicker and/or a less viscous lubricant film atop the ribs will result in a better lubricating effect, but will at the same time cause a larger discharge rate of the lubricating fluid itself. One should note that significant lubricant discharge occurs mainly when the ribs are covered by a relatively thick layer of lubricating fluid. This means that in practice the lubricant film atop the ribs cannot be too thick in order to maintain durability of the lubricating effect. We also remark that in practice, the grooves may have closed ends (instead of open ends as assumed in our model) at the channel entry and exit, and hence the actual discharge rate could be much less than that predicted by the present model. A return current will be in- 


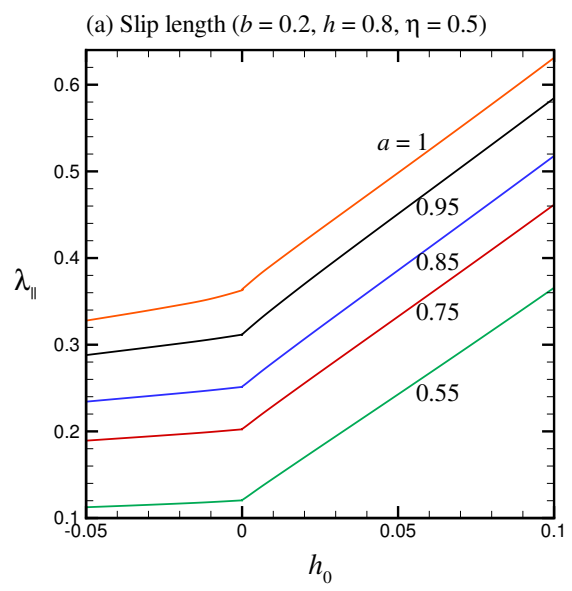

(b) Lubricant flow rate $(b=0.2, h=0.8, \eta=0.5)$

(c) Slip length $(b=0.2, h=0.8, \eta=0.1)$
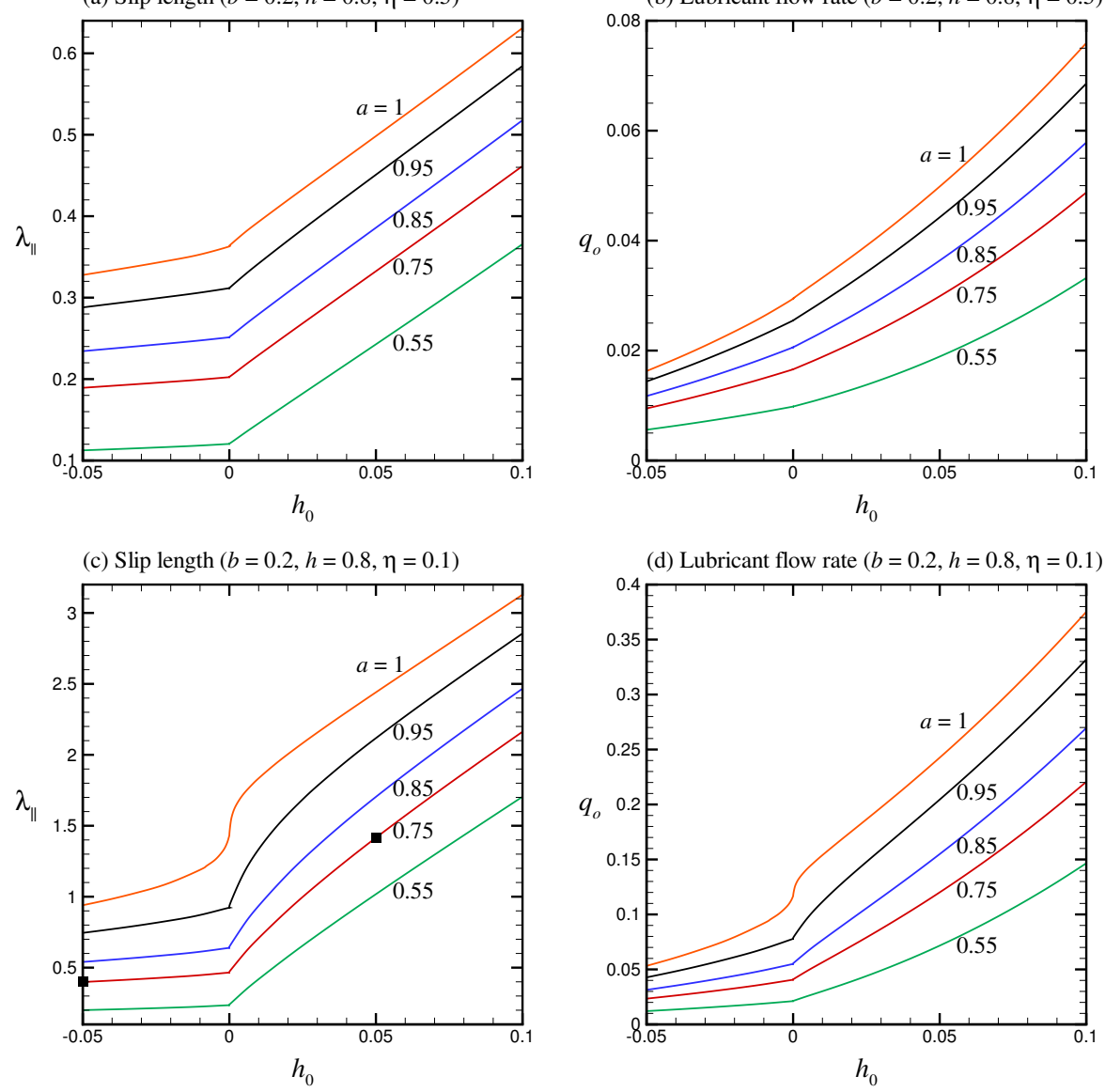

(d) Lubricant flow rate $(b=0.2, h=0.8, \eta=0.1)$

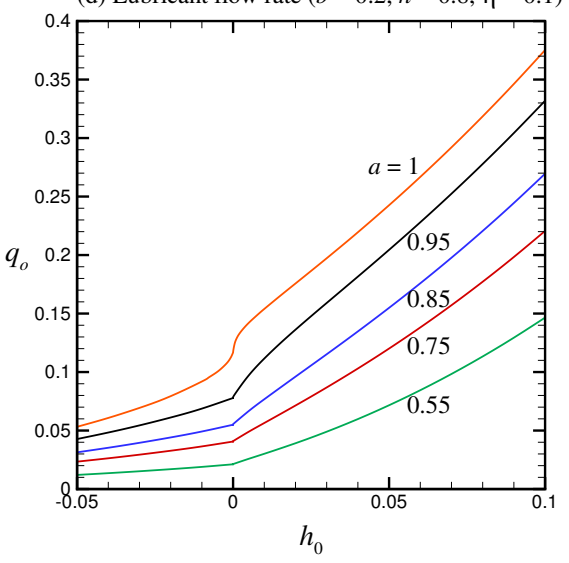

Fig. 3 For longitudinal grooves, the effective slip length $\lambda_{\|}$and lubricant flow rate $q_{o}$ as functions of the lubricant film thickness $h_{0}$ and groove area fraction $a$. The flow fields for the two cases marked by the symbols in (c) are shown in Fig. 4.

duced by a back pressure in the case of closed grooves, which is beyond the scope of the present work. Nevertheless, the qualitative trends revealed here should remain the same irrespective of the end conditions.

To compare the two configurations where the interface is above or below the top of the ribs, we show in Fig. 4 the flow fields for the two cases, which are marked by the squares in Fig. 3(c). This figure confirms that, with a thin lubricating film atop the ribs, the velocity of the working fluid will be materially increased across the entire channel section. Flow enhancement is particularly significant in the region above the ribs. As a result, the working fluid velocity is more uniformly distributed in case (a) than in case (b). Despite different geometries, these longitudinal flow patterns look similar to those presented by Schönecker and Hardt [26] and Schönecker et al. [25]. 

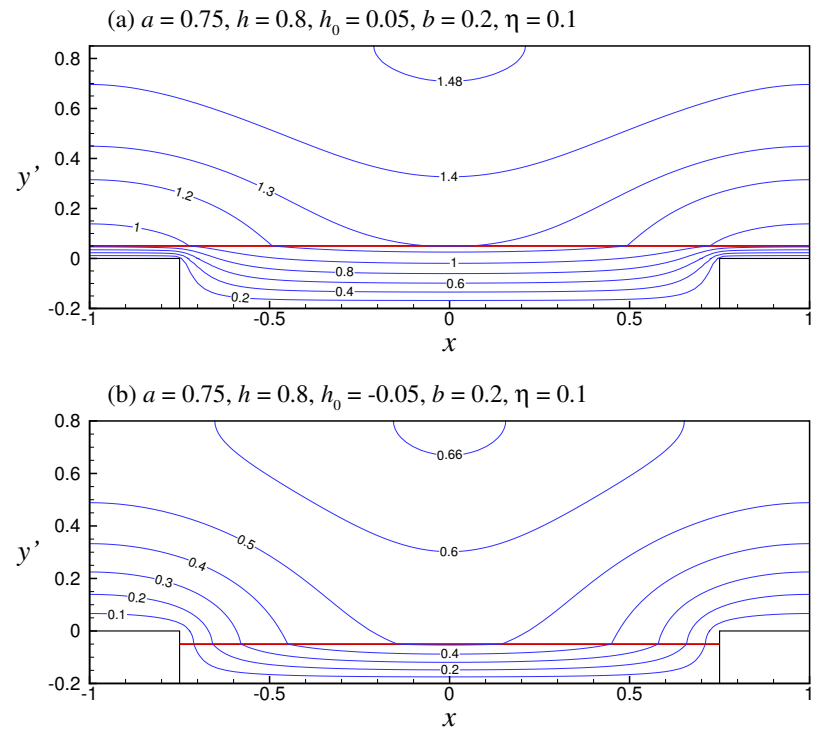

Fig. 4 Velocity isolines for the two cases marked by the symbols in Fig. 3(c). The interface is: (a) above the top of ribs; (b) below the top of ribs.
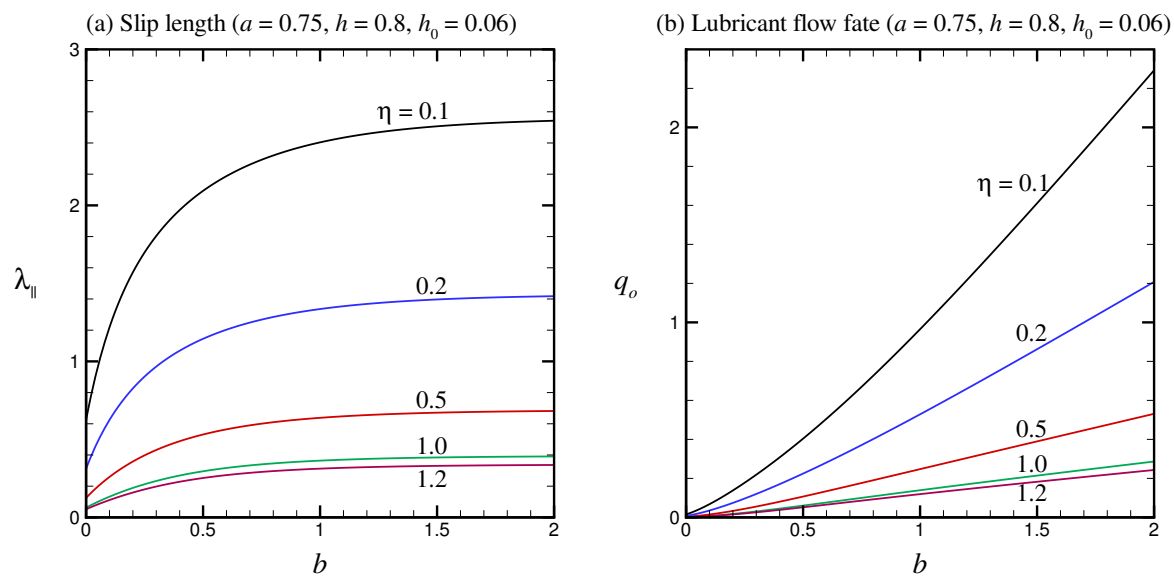

Fig. 5 For longitudinal grooves, the effective slip length $\lambda_{\|}$and lubricant flow rate $q_{o}$ as functions of the groove depth $b$ and viscosity ratio $\eta$. 

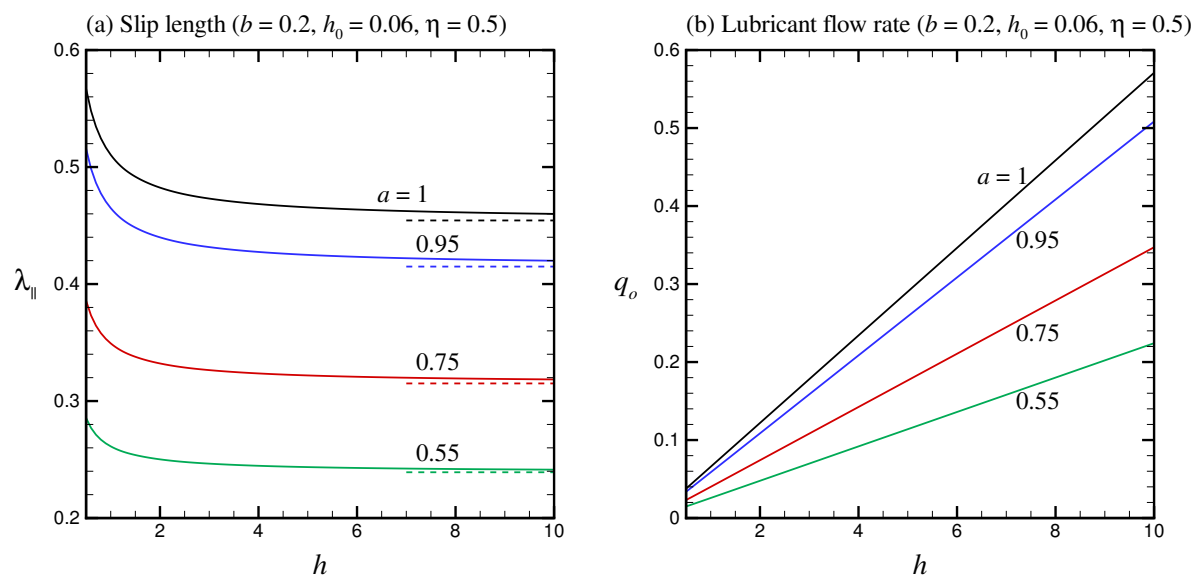

(c) Slip length $\left(b=2, h_{0}=0.06, \eta=0.5\right)$
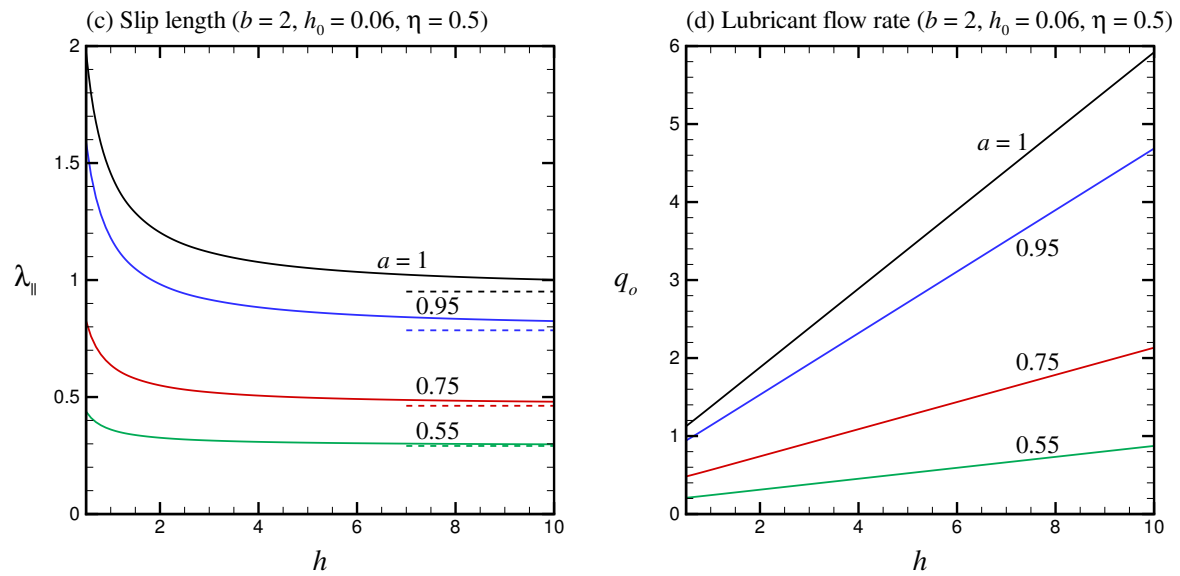

Fig. 6 For longitudinal grooves, the effective slip length $\lambda_{\|}$and lubricant flow rate $q_{o}$ as functions of the working fluid layer thickness $h$ and the groove area fraction $a$. The dashed lines denote the thick-channel limits.

From Fig. 5, we see that the slip length $\lambda_{\|}$and the lubricant flow rate $q_{o}$ both increase monotonically with the groove depth $b$, but with different trends. The rate of increase of $\lambda_{\|}$decreases as $b$ increases such that $\lambda_{\|}$tends to a deep-groove limit for sufficiently large $b$. The deep-groove limit for the slip length can be practically attained, say, when $b=2$ for $\eta=0.5$. In sharp contrast, there is no deep-groove limit for $q_{o}$, which increases without an upper bound as $b$ increases. From this figure, we may infer that it is not beneficial to have excessively deep grooves. One can reason that for too deep grooves, the rate of discharge of the lubricant can be large, while the slip length is already upper-bounded by the deep-groove limit. We further note that at $b=0$ (i.e., zero groove depth or flat wall), the slip length and the lubricant flow rate simplify to $\lambda=\left(h_{0} / \eta\right)\left(1+h_{0} / 2 h\right)$ and $q_{o}=\left(h h_{0}^{2} / 2 \eta\right)\left(1+2 h_{0} / 3 h\right)$. 
We then show in Fig. 6 how the working fluid layer thickness $h$ may have opposite effects on the slip length $\lambda_{\|}$and the lubricant flow rate $q_{o}$. We first see that $\lambda_{\|}$decreases as $h$ increases, but asymptotically tends to a thick-channel limit (the dashes) as $h$ gets sufficiently large. This decreasing trend of effective slip with increasing channel height is true when the longitudinal slip patterns on the two walls are in phase, as has been reported previously by $\mathrm{Ng}$ and Zhou [17] and $\mathrm{Ng}$ and Chen [12]. The thick-channel limit for the slip length can be practically attained when $h=O(1)$ for relatively small $a$ and $b$ (i.e., shallow and narrow grooves), but is not attained until $h \geq O(10)$ for large $a$ and $b$ (i.e., deep and wide grooves). We then see that, in sharp contrast, there is no upper limit for $q_{o}$, which increases linearly as $h$ increases. This is due to the fact that the shear stress increases linearly with distance from the centerline of the channel. Therefore, thicker the layer of the working fluid, larger the shear stress acting on the lubricant. From this figure, we may infer that it is not desirable either to have too thick a channel with longitudinal grooves, which may induce an excessively large rate of discharge of the lubricant.

\section{Transverse grooves}

\subsection{Problem formulation}

We next consider flow through the same lubricant-impregnated-grooved channel, but the grooves are now aligned normal to the principal direction of the flow. The grooves are thereby referred to as transverse grooves. Again, in-phase alignment of grooves on the two walls is assumed, and therefore by symmetry it suffices to consider flow in one half of the channel. A definition sketch of the flow is shown in Fig. 7. The two-dimensional flow, driven by a pressure gradient $K_{x}$ in the $x$-direction, has velocity components $u(x, y)$ and $v(x, y)$ in the $x$ - and $y$-directions. Pressure $p(x, y)$ is also induced as a result of the two-dimensional configuration. Owing to the vertical velocity $v$, a flat interface is not possible; otherwise the condition of zero flux across the interface would be violated. The interface has to deform, as dictated by the flow kinematics, to a curved surface in order to satisfy the condition that the fluid flow velocity on the interface is tangential to the interface. Essentially, the interface will be displaced downward in a manner as shown in Fig. 7. The displacement of the interface is not known a priori but has to be found as part of the solution. This causes the problem not amenable to analytical solution methods. Instead, it has to be solved numerically. The problem here is to solve the continuity, $x$ - and $y$-momentum equations for the three unknowns $(u, v, p)$ as functions of $x$ and $y$.

As in the preceding problem, we shall unless stated otherwise express equations and solutions in terms of non-dimensional quantities. The lengths, velocities, and pressure are, respectively, normalized by $L, K_{x} L^{2} / \mu_{w}$, and $K_{x} L$, where $L$ is half the wall pattern period, $K_{x}=-\partial p / \partial x$ is the axial pressure gradient applied to the fluids, and $\mu_{w}$ is the dynamic viscosity of the working fluid. The ratios of the density and dynamic viscosity of the lubricating fluid $\left(\rho_{o}, \mu_{o}\right)$ to those of the working fluid $\left(\rho_{w}, \mu_{w}\right)$ are denoted by $\gamma=\rho_{o} / \rho_{w}$ and $\eta=\mu_{o} / \mu_{w}$, respectively. 


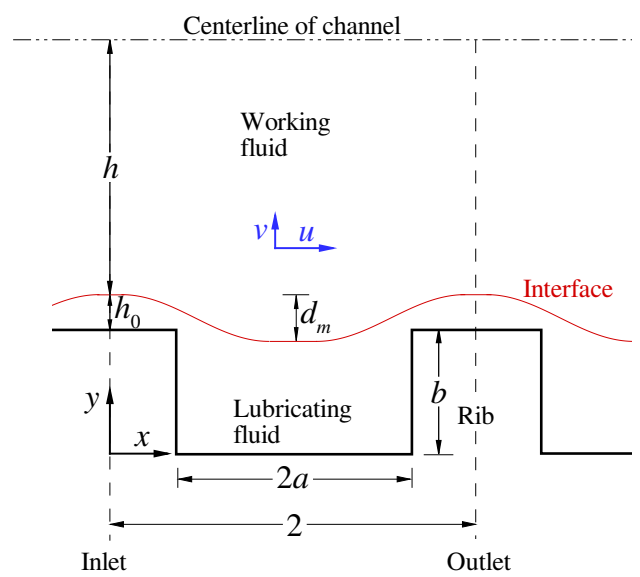

Fig. 7 Definition sketch for flow over transverse grooves, where the flow is driven by a pressure gradient in the $x$-direction.

We consider a computational domain (Fig. 7) that covers one periodic unit of the wall pattern: the inlet boundary is at $x=0$ while the outlet boundary is at $x=2$, both crossing the middle of the respective ribs. Here, the problem has four geometrical quantities as input parameters: $a, b, h$ and $h_{0}$, which are, respectively, half the groove width, the groove depth, half the thickness of the working fluid layer at $x=0$, and the height of the interface above the top of the rib at $x=0$. Note that $a$ also represents the groove area fraction (i.e., the fraction of wall being grooved).

To solve the present problem, which is characterized by the flow of two immiscible fluids separated by a sharp interface, we have developed a numerical scheme based on the front tracking method. The essence of the method is to use a "one-fluid" description for the two-phase flow, which is simulated using the same set of governing equations for the entire domain, where the fluid properties such as density and viscosity may vary as functions of space.

The governing equations are the standard continuity and momentum equations for incompressible Newtonian fluid flow:

$$
\begin{gathered}
\nabla \cdot \mathbf{u}=0, \\
\bar{\rho}\left(\frac{\partial \mathbf{u}}{\partial t}+\mathbf{u} \cdot \nabla \mathbf{u}\right)=-\frac{1}{\operatorname{Re}} \nabla p+\frac{1}{\operatorname{Re}} \nabla \cdot\left[\bar{\mu}\left(\nabla \mathbf{u}+\nabla^{T} \mathbf{u}\right)\right],
\end{gathered}
$$

where $\mathbf{u}=(u, v), t$ is time, Re is the Reynolds number (in terms of physical quantities):

and

$$
\operatorname{Re}=\frac{\rho_{w} U L}{\mu_{w}}, \quad U=\frac{K_{x} L^{2}}{\mu_{w}},
$$

$$
\bar{\rho}=\left\{\begin{array}{l}
1 \text { for working fluid } \\
\gamma \text { for lubricating fluid }
\end{array}, \quad \bar{\mu}=\left\{\begin{array}{l}
1 \text { for working fluid } \\
\eta \text { for lubricating fluid }
\end{array}\right.\right.
$$


In addition to the four geometrical parameters mentioned above, the present problem is controlled also by the following parameters, which characterize the fluid properties and flow dynamics: the density and viscosity ratios, $(\gamma, \eta)$, and the Reynolds number, Re. With the computational domain depicted in Fig. 7, the problem is solved numerically as an initial-boundary-value problem. The two fluids are initially at rest separated by a flat interface located at a height $h_{0}$ above the top of the rib/groove. The flow is driven from $t>0$ onward by a steady pressure gradient. On the outlet $x=2$, the pressure is fixed at 0 , while on the inlet $x=0$, the pressure is fixed at 2 in order to impose a net unity pressure gradient on the fluids. Also, periodic conditions are prescribed on the inlet and outlet for the velocities, and therefore $\partial u / \partial x$ and $v$ are both zero on the inlet/outlet. Symmetry is to be satisfied along the centerline of the channel. Hence, along the top of the domain, the condition of zero shear stress is imposed. No-slip condition is specified on the solid walls. The computation proceeds with time until the steady state is practically achieved. In each time advancement, the interface is kept at a fixed height of $h_{0}$ atop the rib at the inlet/outlet, while between the inlet and outlet it may move as dictated by the instantaneous flow kinematics and dynamics. The downward movement of the interface will reduce the amount of lubricating fluid in the system with time. When the steady state is reached, the interface will become a stationary curved surface that satisfies the kinematic and dynamic boundary conditions. The tracking of the movement of the interface is an essential part of the numerical solution. The key features about the front tracking method that we have used are briefly described in the appendix.

Again, the overall lubricating effect is to be represented by an effective slip length, which can be inferred from a macroscopic relationship between flow rate and the applied pressure gradient. As in the preceding problem, on matching with the rate of flow through an equivalent slit channel of height $2 h$ bounded by plane walls of slip length $\lambda: q=2 h^{3} / 3+2 h^{2} \lambda$, we may obtain the effective slip length for transverse grooves as follows:

$$
\lambda_{\perp}=\left(\frac{q_{w}-q_{\mathrm{ns}}}{q_{\mathrm{ns}}}\right) \frac{h}{3}
$$

where $q_{\mathrm{ns}}=2 h^{3} / 3$ is the flow rate for the base case of no-slip walls, and

$$
q_{w}=2 \int_{h_{0}}^{h+h_{0}} u_{w} \mathrm{~d} y^{\prime} \quad \text { at } x=0
$$

is the flow rate of the working fluid under the lubricating effect, where $y^{\prime}=y-b$. The rate of flow of the lubricating fluid is also found as follows:

$$
q_{o}=\int_{0}^{h_{0}} u_{o} \mathrm{~d} y^{\prime} \quad \text { at } x=0 .
$$

We have used Simpson's formula to evaluate the above integrals numerically.

\subsection{Results and discussion}

We have generated results based on a uniform staggered Cartesian grid with a grid spacing of 0.02 , and a time step of $O\left(10^{-4}\right)$. It normally takes $O\left(10^{5}\right)$ time steps to 
reach a steady state. Our primary objective is to determine the effective slip length $\lambda_{\perp}$ and the lubricant flow rate $q_{o}$ as functions of the following parameters: $a$ (area fraction of the groove), $b$ (groove depth), $h_{0}$ (thickness of the lubricant film atop the rib at $x=0$ ), $h$ (half the thickness of the working fluid layer at $x=0$ ), and $\eta$ (viscosity ratio). Except for the accuracy checking of the scheme, the values of $\gamma=$ 0.8 and $\operatorname{Re}=1$ have been used for the density ratio and the Reynolds number for the numerical results presented below. We do not vary these two parameters, as the inertia effect is not considered a significant factor in the present problem. In fact, we have generated results for different $\mathrm{Re}$, and found that the results are nearly independent of the Reynolds number as long as $\operatorname{Re} \leq O(1)$; see Table 1 for some sample results.

Table 1 Effect of Re on the working fluid flow rate $q_{w}$ and effective slip length $\lambda_{\perp}$ for transverse grooves, where $a=0.75, b=0.22, h=0.8, h_{0}=0.06, \eta=0.2$.

\begin{tabular}{c|cccc}
\hline $\operatorname{Re}$ & 1 & 0.5 & 0.2 & 0.1 \\
\hline$q_{w}$ & 0.7856 & 0.7854 & 0.7848 & 0.7839 \\
$\lambda_{\perp}$ & 0.347084 & 0.346927 & 0.346459 & 0.345755 \\
\hline
\end{tabular}

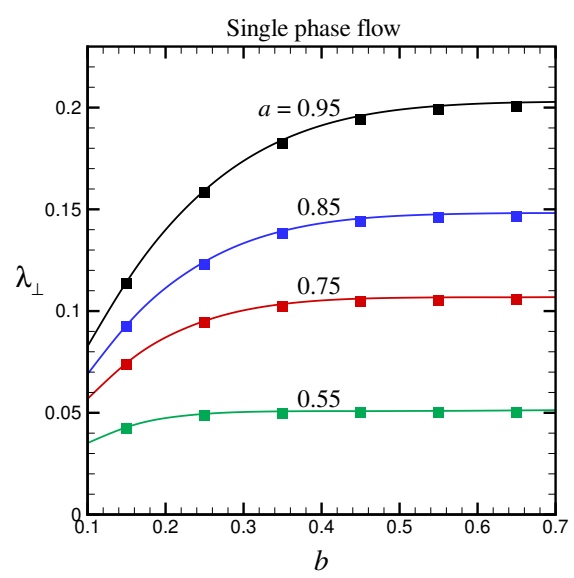

Fig. 8 For transverse grooves, the effective slip length $\lambda_{\perp}$ as a function of groove depth $b$ and groove area fraction $a$, as computed by the numerical model (lines), and by the analytical model (symbols).

Let us first check the accuracy of our numerical scheme by comparing results for the special case of low-Reynolds-number flow of a single fluid (i.e., $\gamma=\eta=1$, $\mathrm{Re} \ll 1)$ through a channel with transverse grooves. For this special case, the problem can be solved analytically using, again, the methods of domain decomposition and eigenfunction expansion [13-15,32]. The comparison, shown in Fig. 8, reveals that the slip length $\lambda_{\perp}$ found as a function of $a$ and $b$ by the numerical model agrees very 
well with that by the analytical model. The difference is less than $0.1 \%$. This provides a support to the accuracy of our numerical model.
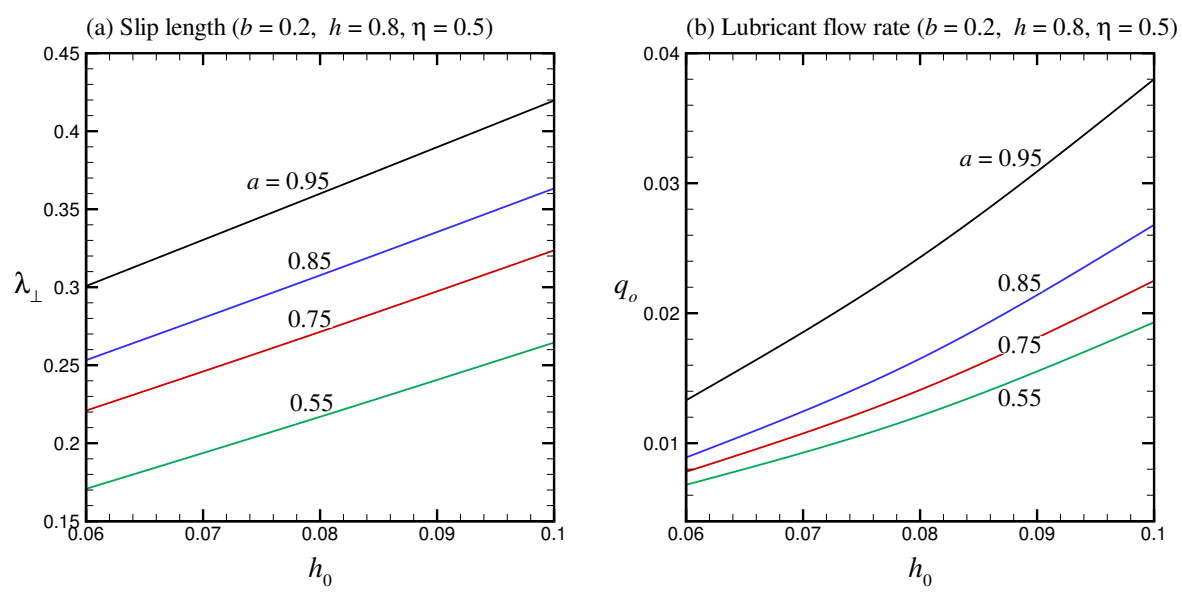

Fig. 9 For transverse grooves, the effective slip length $\lambda_{\perp}$ and lubricant flow rate $q_{o}$ as functions of the lubricant film thickness $h_{0}$ and groove area fraction $a$.

We first show in Fig. 9 how the slip length and lubricant flow rate may change as functions of the lubricant film thickness and the groove area fraction. The fashions in which $\lambda_{\perp}$ and $q_{o}$ increase with increasing $a$ and $h_{0}$ are qualitatively the same as those shown earlier in Fig. 3, the counterpart for longitudinal grooves. On inspecting the values, one will find that, with the same set of parameters, the slip length is in general smaller for transverse grooves than for longitudinal grooves. The same statement applies to the lubricant flow rate. This quantitative difference between longitudinal and transverse grooves is somewhat expected. It is well known in the literature that the effective slip for flow normal to a two-dimensional wall pattern (e.g., alternate stick-slip slots) is smaller than that for flow parallel to the pattern; see, e.g., Refs. [16, 20,32]. We provide in Table 2 some values of the slip length and lubricant flow rate for the cases of longitudinal and transverse grooves, as have been shown in Figs. 3 and 9.

We next show in Fig. 10 the slip length $\lambda_{\perp}$, the lubricant flow rate $q_{o}$, as well as the peak depression of the interface $d_{m}$, as functions of the the groove depth $b$. Depending on the viscosity ratio $\eta$, the trends can be dramatically different from those shown earlier in Fig. 5 for the case of longitudinal grooves. It is remarkable that, for a small viscosity ratio, say $\eta \leq 0.1, \lambda_{\perp}$ and $q_{o}$ will vary in a non-monotonic manner with the groove depth $b$. Figure 10(a) shows that, for $\eta=0.1,0.05$, the slip length $\lambda_{\perp}$ first decreases as $b$ increases, reaching a minimum before increasing to approach a deep-groove limit at large $b$. One can reason that this decrease of the slip length is associated with an increased downward displacement of the interface above the groove. Figure 10(c) shows that the peak interface depression $d_{m}$, which happens at $x=1$ 
Table 2 Comparison of slip length $\lambda$ and lubricant flow rate $q_{o}$ for longitudinal and transverse grooves, where $b=0.2, h=0.8, \eta=0.5$.

\begin{tabular}{c|c|cc|cc}
\hline$a$ & $h_{0}$ & \multicolumn{2}{c|}{ Longitudinal grooves } & \multicolumn{2}{c}{ Transverse grooves } \\
\cline { 3 - 6 } & & $\lambda_{\|}$ & $q_{o}$ & $\lambda_{\perp}$ & $q_{o}$ \\
\hline 0.95 & 0.06 & 0.4775 & 0.0486 & 0.3008 & 0.0133 \\
& 0.08 & 0.5308 & 0.0581 & 0.3600 & 0.0243 \\
& 0.1 & 0.5845 & 0.0686 & 0.4197 & 0.0380 \\
\hline 0.75 & 0.06 & 0.3581 & 0.0332 & 0.2209 & 0.0078 \\
& 0.08 & 0.4096 & 0.0405 & 0.2714 & 0.0141 \\
& 0.1 & 0.4616 & 0.0488 & 0.3236 & 0.0225 \\
\hline
\end{tabular}

(a) Slip length ( $\left.a=0.75, h=0.8, h_{0}=0.06\right)$

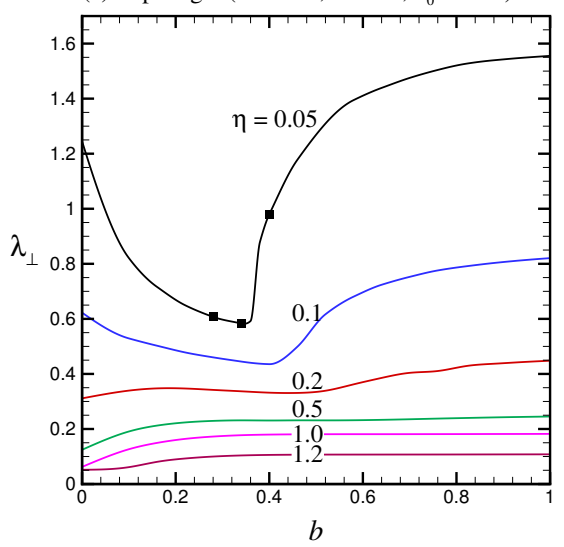

(b) Lubricant flow rate $\left(a=0.75, h=0.8, h_{0}=0.06\right)$

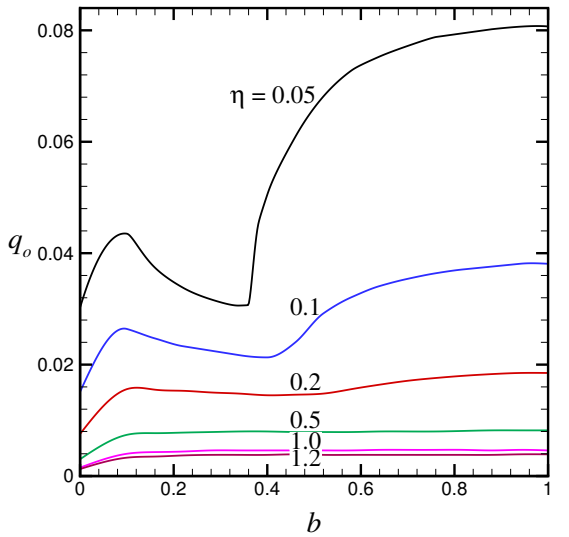

(c) Peak interface depression $\left(a=0.75, h=0.8, h_{0}=0.06\right)$

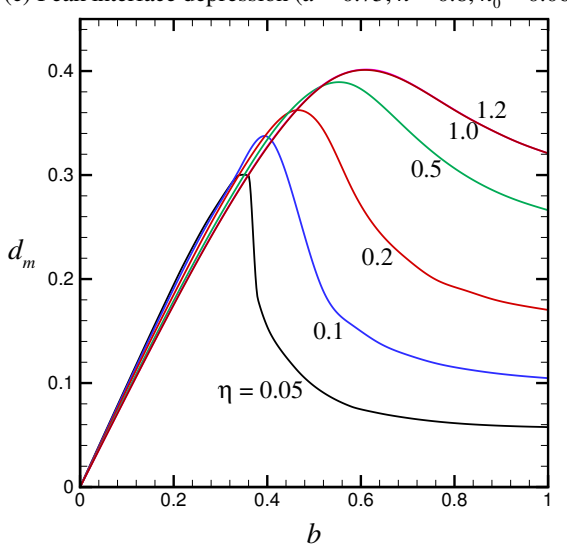

Fig. 10 For transverse grooves, the effective slip length $\lambda_{\perp}$, lubricant flow rate $q_{o}$, and peak interface depression $d_{m}$, as functions of the groove depth $b$ and viscosity ratio $\eta$. The flow fields for the three cases marked by the symbols in (a) are shown in Fig. 11. 
(i.e., the center of the groove), first increases as the groove depth $b$ increases, reaching a maximum before falling off sharply to approach a deep-groove limit at large $b$. A larger depression or undulation of the interface amounts to a rougher boundary as far as the working fluid flow is concerned. This translates to a higher flow resistance, or a smaller effective slip, for flow over a deeper transverse groove. This happens until the groove depth attains the point where the undulation of the interface reaches the maximum. Beyond this point, the interface undulation falls off, giving rise to a smoother boundary (or a larger effective slip) for the working fluid flow. Figure 10(b) shows that the corresponding $q_{o}$ also reaches a minimum at the point where the slip length is the minimum. In short, we may ascribe these non-monotonic variations of the slip length and lubricant flow rate to the extent of downward displacement of the interface into the groove as the groove depth varies. Such non-monotonic variations are distinct features for flow over transverse grooves. For flow over longitudinal grooves, the interface will always be flat unaffected by the groove depth.

Figures $10(a, b)$ also show that, for a sufficiently large viscosity ratio, say $\eta \geq 0.5$, the non-monotonic variations mentioned above will disappear. Both the slip length and the lubricant flow rate will increase monotonically as the groove depth increases, approaching some finite limits at large groove depth. This happens when the lubricant viscosity is sufficiently high, for which the roughness effect arising from the interface undulation is much less influential. On comparing Fig. 10 with Fig. 5, one can readily see that the slip length and lubricant flow rate for the case of transverse grooves are both smaller than the counterparts for the case of longitudinal grooves. We provide in Table 3 some values of the slip length and lubricant flow rate for the cases of longitudinal and transverse grooves, as have been shown in Figs. 5 and 10.

Table 3 Comparison of slip length $\lambda$ and lubricant flow rate $q_{o}$ for longitudinal and transverse grooves, where $a=0.75, h=0.8, h_{0}=0.06$.

\begin{tabular}{c|c|cc|cc}
\hline$\eta$ & $b$ & \multicolumn{2}{|c|}{ Longitudinal grooves } & \multicolumn{2}{c}{ Transverse grooves } \\
\cline { 3 - 6 } & & $\lambda_{\|}$ & $q_{o}$ & $\lambda_{\perp}$ & $q_{o}$ \\
\hline 0.1 & 0.1 & 1.2182 & 0.0677 & 0.5290 & 0.0264 \\
& 0.4 & 1.9700 & 0.3079 & 0.4355 & 0.0213 \\
& 1.0 & 2.4040 & 0.9650 & 0.8206 & 0.0381 \\
\hline 0.2 & 0.1 & 0.6245 & 0.0352 & 0.3394 & 0.0156 \\
& 0.4 & 1.0685 & 0.1706 & 0.3319 & 0.0145 \\
& 1.0 & 1.3355 & 0.5286 & 0.4480 & 0.0185 \\
\hline
\end{tabular}

For illustration, we show in Fig. 11 the flow fields for the three cases that are marked by a symbol in Fig. 10(a), where $\eta=0.05$. This figure clearly shows how the interface may be displaced downward to a different extent into the groove depending on the groove depth. For a relatively shallow groove, the interface may sag so substantially at the center that it can almost reach the bottom of the groove; see Fig. 11(a). Two isolated vortices are formed at the two corners of the groove. At the point when the interface displacement at the center is the maximum, the interface may penetrate as much as two-thirds of the groove depth; see Fig. 11(b). For flow over a deeper groove, the interface will, to the contrary, be displaced less into the 

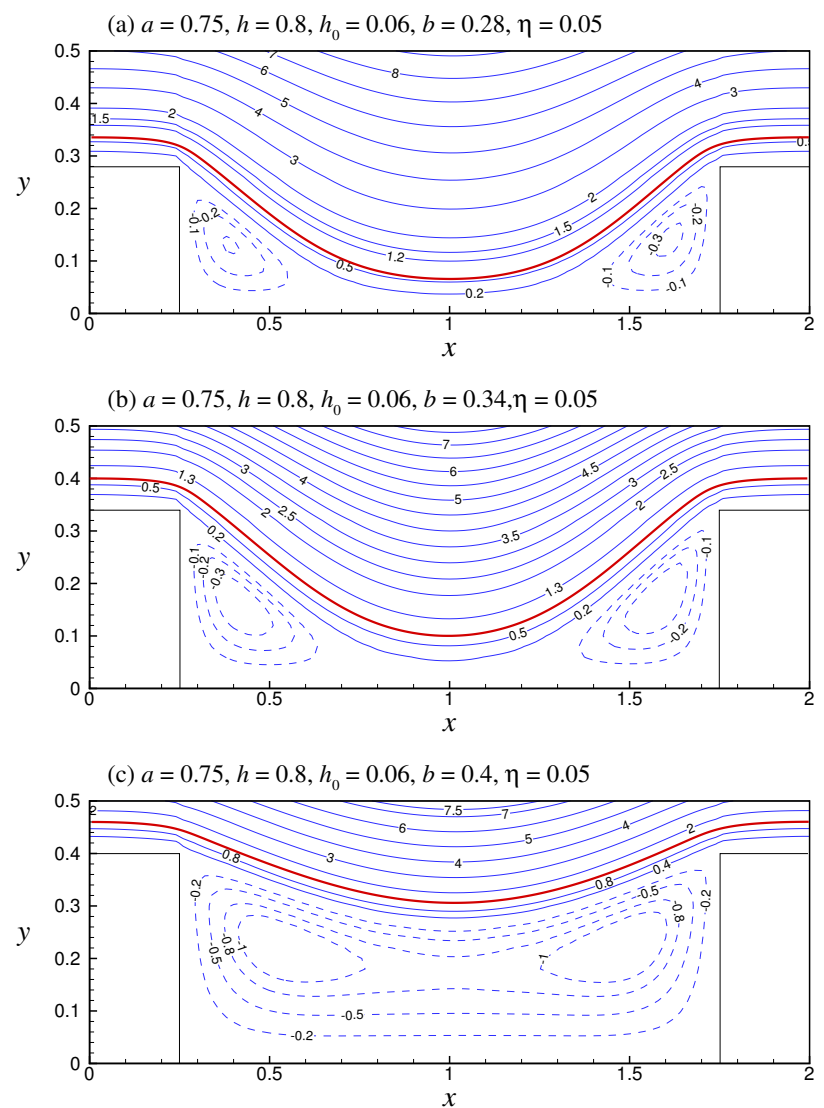

Fig. 11 Streamlines for the three cases marked by the symbols in Fig. 10(a).

groove, resulting in a smoother flow of the working fluid over the groove. This happens when the two vortices grow in size and intensity, and gradually merge to form a single recirculating zone in the groove. As can be seen in this figure, the net flow of the lubricant is limited to a thin layer underneath the interface. This can be called a skimming flow of the lubricant. Much of the lubricant flow inside the groove is in the form of a recirculating cell. Energy is extracted from the main stream flow to maintain the vortical flow of the lubricant. This explains why the drag is higher for flow over transverse grooves than that over longitudinal grooves.

We finally show in Fig. 12 how the working fluid layer thickness $h$ may have opposite effects on the slip length $\lambda_{\perp}$ and the lubricant flow rate $q_{o}$. The trends are similar to those seen earlier in Fig. 6 for the case of longitudinal grooves. First, the transverse effective slip length $\lambda_{\perp}$ decreases, while the lubricant flow rate increases, as the working fluid layer thickness $h$ increases. Second, at sufficiently large $h, \lambda_{\perp}$ will asymptotically tend to a thick-channel limit (not shown in the figure), while $q_{o}$ may increase in a nearly linear manner without an upper bound. Third, on comparing 

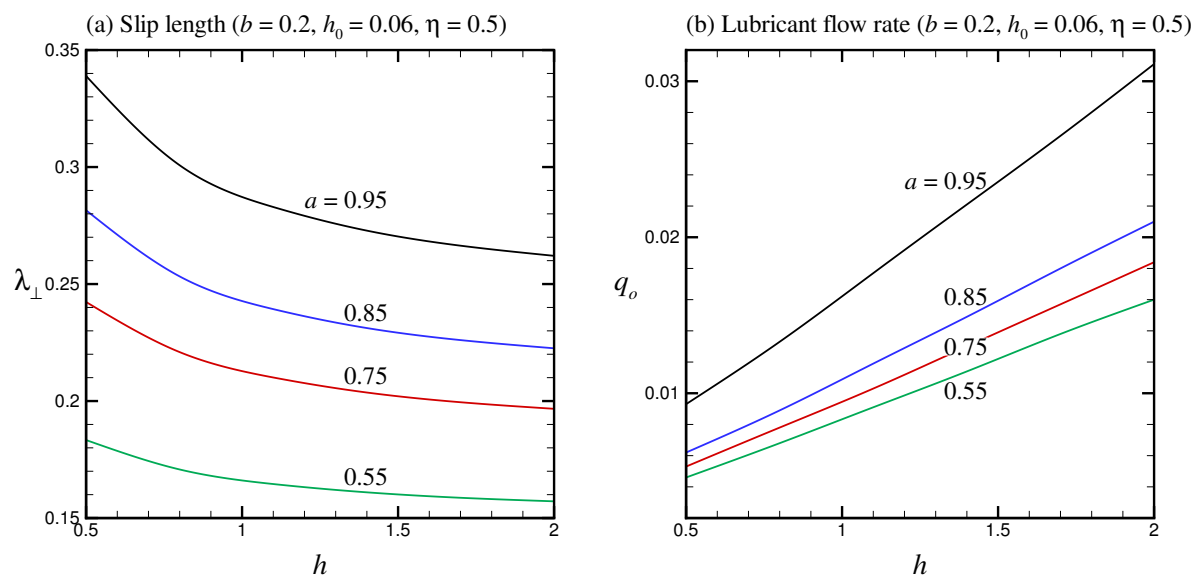

Fig. 12 For transverse grooves, the effective slip length $\lambda_{\perp}$ and lubricant flow rate $q_{o}$ as functions of the working fluid layer thickness $h$ and the groove area fraction $a$.

Figs. 4 and 10, it is found again that, with the same set of parameters, $\lambda_{\perp}$ is smaller than $\lambda_{\|}$, and $q_{o}$ is also smaller for transverse grooves than for longitudinal grooves. From Figs. 4 and 10, we may remark again that it is not desirable to use a grooved surface to lubricate flow in a too deep channel, whether the flow is parallel or normal to the grooves. Otherwise, the rate of discharge of the lubricating fluid can be rather large. We provide in Table 4 some values of the slip length and lubricant flow rate for the cases of longitudinal and transverse grooves, as have been shown in Figs. 6 and 12.

Table 4 Comparison of slip length $\lambda$ and lubricant flow rate $q_{o}$ for longitudinal and transverse grooves, where $b=0.2, h_{0}=0.06, \eta=0.5$.

\begin{tabular}{c|c|cc|cc}
\hline$a$ & $h$ & \multicolumn{2}{c|}{ Longitudinal grooves } & \multicolumn{2}{c}{ Transverse grooves } \\
\cline { 3 - 6 } & & $\lambda_{\|}$ & $q_{o}$ & $\lambda_{\perp}$ & $q_{o}$ \\
\hline 0.95 & 0.5 & 0.5158 & 0.0337 & 0.3389 & 0.0093 \\
& 0.8 & 0.4775 & 0.0486 & 0.3008 & 0.0133 \\
& 2.0 & 0.4399 & 0.1086 & 0.2621 & 0.0311 \\
\hline 0.75 & 0.5 & 0.3858 & 0.0231 & 0.2423 & 0.0053 \\
& 0.8 & 0.3581 & 0.0332 & 0.2209 & 0.0078 \\
& 2.0 & 0.3321 & 0.0741 & 0.1967 & 0.0184 \\
\hline
\end{tabular}

\section{Concluding remarks}

We have investigated longitudinal and transverse flows through a slit channel bounded by slippery surfaces that are microstructured with two-dimensional periodic rectangular grooves infused with a lubricant. While the problem for longitudinal flow is 
solved analytically using the methods of domain decomposition and eigenfunction expansion, the problem for transverse flow is solved numerically using the front tracking method in order to compute the undetermined interface between the working and lubricating fluids.

The key findings of the present study are summarized as follows. First, for wallbounded pressure-driven flow, the effective slip depends strongly on the geometry of the underlying microtextures. This is in sharp contrast to what has been found for the mobility of droplets on LIS, which is essentially insensitive to the geometry of the underlying substrate. We have shown how the effective slip may vary depending on the width and depth of the grooves, the channel height, and the direction of flow relative to the grooves. Second, the position of the interface, whether above or below the top of the ribs/grooves, is particularly influential in determining the effective slip that can be derived from the lubricant infused surface. The effective slip length can be several times larger when the lubricant overfills the grooves to form a film atop the ribs than when the lubricant is completely confined inside the grooves. This is consistent with the observation made by Wong et al. [35] that liquid repellency is primarily due to the lubricating film covering the textures. Third, lubricant-impregnated grooves that are aligned parallel to the flow may in general give rise to higher effective slip than when the grooves are aligned perpendicular to the flow. These two limiting alignments should correspond to the upper and lower bounds for the effective slip obtainable from the grooved surface. Fourth, in the case of transverse grooves, the downward displacement of the interface above a groove may vary non-monotonically with the groove depth. There exists a particular groove depth at which the interface undulates with the maximum amplitude, corresponding to the roughest boundary for the working fluid flow, thereby the minimum effective slip. Fifth, for transverse grooves, the forward flow of the lubricant is a thin layer skimming over the grooves, while much of the flow inside the grooves is in the form of recirculating cells. As a result, the net lubricant flow over transverse grooves is limited. In sharp contrast, for longitudinal grooves, the entire body of the lubricant flows forward, resulting in a possibly large discharge of the lubricant over time.

It is desirable to study in the future other effects that may also control the performance of lubricant-impregnated surfaces when used to provide drag reduction in wall-bounded flows. We may consider, for examples, advanced effects due to threedimensional textures (such as arrays of posts on a square lattice), thermocapillary, high Reynolds number, and so on. In the present study, we have ignored pressure difference across the fluid-fluid interface arising from interfacial tension. In a future study, this assumption can be relaxed, thereby allowing the interface to deform according to the Laplace pressure condition [33]. The problem would be challenging as the deformation of the interface is completely unknown, and it is of fundamental interest to find out how this will affect the effective slip length. Another issue that deserves investigation is the possible hydrodynamic instability of the lubricant film atop the structured surface.

Acknowledgements The authors are grateful to the two anonymous reviewers for their comments and suggestions, which have helped improve the writing of the paper. Financial support was given by the Re- 
search Grants Council of the Hong Kong Special Administrative Region, China, through General Research Fund Project No. 17206615.

\section{Appendix: Numerical scheme based on the front tracking method}

In this appendix, we provide some basic details about the front tracking method that we have adopted to solve the present problem. For further details about the method, see Tryggvason et al. [31].

The method is essentially to solve the governing equations by the finite volume approach. Accordingly, we discretize the computational domain into sufficiently small regular control volumes, and approximate the variables such as velocity, pressure, and density using their average values over each control volume. As in standard finite volume methods, a staggered mesh is used, where the scalar variables including pressure, density, and dynamic viscosity are stored in the cell center of the control volume while the velocities are located on the respective cell faces. The projection method is used to solve the momentum equation. We first compute the velocity field without considering the pressure, and get a temporary non-divergence-free velocity field $\mathbf{u}^{*}$ :

$$
\bar{\rho}^{n}\left(\frac{\mathbf{u}^{*}-\mathbf{u}^{n}}{\Delta t}+\mathbf{u}^{n} \cdot \nabla \mathbf{u}^{n}\right)=\frac{1}{\operatorname{Re}} \nabla \cdot\left[\bar{\mu}^{n}\left(\nabla \mathbf{u}^{n}+\nabla^{T} \mathbf{u}^{n}\right)\right],
$$

where $n$ denotes the old time level. The pressure $p^{n}$ is then added, which will update the velocity to the new time level:

$$
\bar{\rho}^{n}\left(\frac{\mathbf{u}^{n+1}-\mathbf{u}^{*}}{\Delta t}\right)=-\frac{1}{\operatorname{Re}} \nabla p^{n} .
$$

Using the requirement that the velocity is divergence-free at the new time level: $\nabla$. $\mathbf{u}^{n+1}=0$, we may take the divergence of the equation above and get the following governing equation for $p^{n}$ :

$$
\nabla \cdot\left(\frac{1}{\bar{\rho}^{n}} \nabla p^{n}\right)=\frac{\operatorname{Re}}{\Delta t} \nabla \cdot \mathbf{u}^{*}
$$

Since the density ratio in our computation does not deviate much from 1 , this Poisson equation for the pressure can be solved efficiently using the successive over relaxation (SOR) method.

In the present problem, where the Reynolds number is not too large, the advection and diffusion terms can be accurately computed using the centered differencing scheme. For high Reynolds numbers, we may use either the QUICK (Quadratic Upstream Interpolation for Convective Kinematics) or ENO (Essentially Non-Oscillating) schemes to compute the advection terms.

To keep track of the movement of the interface, the front tracking method is used. The interface between the working and lubricating fluids is represented by a set of linked points. The lines between two adjacent points are called elements. As these front points do not necessarily coincide with the fixed grid points, we need to determine the position relationship between these two sets of points. Since the fixed grid 
points are positioned on a regular lattice, we may readily find the grid point that is closest to a front point and establish the position relationship. The use of the position relationship is discussed in detail below.

The solutions to the continuity and momentum equations are sought at discrete points that make up the fixed grid. This makes it necessary to pass on the value of a quantity on the front points to the fixed grid points. On passing on the value, it is important to maintain the conservation of the quantity. In two-dimensional problems, we may use $\phi_{f}$ to denote the value of a quantity on the interface in units per length, and $\phi_{i, j}$ to represent the value of the quantity at the fixed grid point $(i, j)$ in units per control volume. For any conserved quantity $\phi$, we have

$$
\int_{\Delta V} \phi_{i, j} \mathrm{~d} V=\int_{\Delta s} \phi_{f}(s) \mathrm{d} s
$$

where $\Delta s$ is the length of the interface that is smoothed to the fixed grid area $\Delta V$. The discrete form of the grid value is therefore

$$
\phi_{i, j}=\sum_{l} \phi_{f}^{l} w_{i, j}^{l} \frac{\Delta s l}{\Delta x \Delta y}
$$

where $\Delta s l$ is the length of the element $l$ and $\Delta x$ and $\Delta y$ are the grid spacings. The weighting function $w_{i, j}^{l}$ represents the fraction each grid point gets from the front and the sum of the weighting functions for every element is 1 . In two dimensions, the weighting for a fixed grid point $(x, y)$ from a front point $\mathbf{x}_{f}=\left(x_{f}, y_{f}\right)$ can be written as

$$
w_{i, j}^{l}\left(\mathbf{x}_{f}\right)=d\left(x_{f}-i \Delta x\right) d\left(y_{f}-j \Delta y\right)
$$

In our numerical model, we use equal grid spacings $\Delta x=\Delta y$, and we may apply the Peskin weighting function [19] as follows:

$$
d(r)= \begin{cases}{\left[1+\cos \left(\frac{\pi r}{2 \Delta x}\right)\right] /(4 \Delta x)} & |r|<2 \Delta x \\ 0 & |r| \geq 2 \Delta x\end{cases}
$$

The movement of the interface requires a reconstruction of the fields of density and other fluid properties. In our method, density is taken to be a marker function. At each time step, we first reconstruct the density field, and then use the density to deduce the values of other properties. The density can be expressed as

$$
\bar{\rho}=\mathrm{H}+(1-\mathrm{H}) \gamma,
$$

where $\mathrm{H}$ is the Heaviside step function which equals 1 at points occupied by the working fluid and equals 0 at points occupied by the lubricant. In the front tracking method, the interface can mark the density jump. We translate the sharp density jump across the interface into a steep gradient on the fixed grid. The density gradient can be related to the jump as below:

$$
\nabla \bar{\rho}=(1-\gamma) \nabla \mathrm{H}=\Delta \rho \delta(n) \mathbf{n},
$$

where $\Delta \rho=1-\gamma$ is the density jump across the interface, $\delta$ is the Dirac delta function, and $\mathbf{n}$ is the unit normal to the interface (pointing toward the working fluid). 
Here, use has been made of the relation $\nabla \mathrm{H}=\delta(n) \mathbf{n}$. The front is now represented by a $\delta$-function. Accordingly, we can smooth the density jump by assigning values on the fixed grid. Using the above equation, we can get the density gradient equation

$$
\int_{V} \nabla \bar{\rho} \mathrm{d} V=\int_{S_{I}} \Delta \rho \mathbf{n} \mathrm{d} s
$$

where $V$ represents the control volume, and $S_{I}$ is the front length in the control volume.

For the density value at $(i, j)$, once we have obtained the density gradient on the staggered grid, we can integrate the density gradient from the surrounding four points and average the value to get a relatively accurate density field. Other fluid property fields can then be reconstructed easily using a linear mapping between the fluid property and the density. All the updating of the fluid properties are limited to points near the interface in our numerical method.

Since points on the interface move with different velocities, the interface will deform as time advances. As a result, after some time, the distribution of points becomes sparse on some parts of the interface, but crowded on other parts. To accurately represent the interface, we have to check, and adjust, if needed, the location of the front points at the end of each time step. In two-dimensional problems, we define a maximum and a minimum distance. Then we compare the distance between two arbitrary adjacent front points with the pre-defined maximum and minimum value. If the distance is larger than the maximum value, we add a new point between these two points. In a similar way, if the distance is smaller than the minimum value, we delete a point and continue computing the distance between the undeleted point and the point next to the deleted point. By adding and deleting points, we can maintain relatively accurate distribution of points on the interface. In general, three to four front points per control volume is sufficient for a good representation of the interface.

The boundary conditions are imposed on the grid as follows. As the velocities are stored on the respective cell faces, we can directly set the normal velocity equal to what it should be on the boundary. In order to specify the tangential velocities easily, we add one row of ghost cells immediately outside the boundary. Then the tangential velocities can be obtained using a linear interpolation between the velocities inside the wall and the ghost velocities. In addition, periodic boundary conditions for the velocities are to be satisfied on the inlet and outlet. Suppose the whole domain is discretized by $N$ cells in the $x$ direction. Taking the ghost points into consideration, we have $N+2$ points in one row. Then we can easily implement the periodic boundary using the following equations:

$$
\mathbf{u}(1, j)=\mathbf{u}(N+1, j) \quad \text { and } \quad \mathbf{u}(N+2, j)=\mathbf{u}(2, j) .
$$

\section{References}

1. Anand, S., Paxson, A.T., Dhiman, R., Smith, J.D., Varanasi, K.K.: Enhanced condensation on lubricant-impregnated nanotextured surfaces. ACS Nano 6(11), 10,122-10,129 (2012) 
2. Bohn, H.F., Federle, W.: Insect aquaplaning: Nepenthes pitcher plants capture prey with the peristome, a fully wettable water-lubricated anisotropic surface. Proceedings of the National Academy of Sciences of the United States of America 101(39), 14,138-14,143 (2004)

3. Carlson, A., Kim, P., Amberg, G., Stone, H.A.: Short and long time drop dynamics on lubricated substrates. EPL 104(3), 34,008 (2013)

4. Eifert, A., Paulssen, D., Varanakkottu, S.N., Baier, T., Hardt, S.: Simple fabrication of robust water-repellent surfaces with low contact-angle hysteresis based on impregnation. Advanced Materials Interfaces 1(3), 1300,138 (2014)

5. Epstein, A.K., Wong, T.S., Belisle, R.A., Boggs, E.M., Aizenberg, J.: Liquidinfused structured surfaces with exceptional anti-biofouling performance. Proceedings of the National Academy of Sciences 109(33), 13,182-13,187 (2012)

6. Huang, X., Chrisman, J.D., Zacharia, N.S.: Omniphobic slippery coatings based on lubricant-infused porous polyelectrolyte multilayers. ACS Macro Letters 2(9), 826-829 (2013)

7. Kim, P., Wong, T.S., Alvarenga, J., Kreder, M.J., Adorno-Martinez, W.E., Aizenberg, J.: Liquid-infused nanostructured surfaces with extreme anti-ice and antifrost performance. ACS Nano 6(8), 6569-6577 (2012)

8. Lafuma, A., Quéré, D.: Slippery pre-suffused surfaces. EPL 96(5), 56,001 (2011)

9. Lalia, B.S., Anand, S., Varanasi, K.K., Hashaikeh, R.: Fog-harvesting potential of lubricant-impregnated electrospun nanomats. Langmuir 29(42), 13,08113,088 (2013)

10. Li, J., Kleintschek, T., Rieder, A., Cheng, Y., Baumbach, T., Obst, U., Schwartz, T., Levkin, P.A.: Hydrophobic liquid-infused porous polymer surfaces for antibacterial applications. ACS Applied Materials \& Interfaces 5(14), 6704-6711 (2013)

11. Liu, H., Zhang, P., Liu, M., Wang, S., Jiang, L.: Organogel-based thin films for self-cleaning on various surfaces. Advanced Materials 25(32), 4477-4481 (2013)

12. Ng, C.O., Chen, B.: Microchannel flows with superhydrophobic surfaces: Effects of phase shift of wall patterns. In: Proceedings of the 14th Asian Congress of Fluid Mechanics, pp. 1037-1041 (2013)

13. Ng, C.O., Chu, H.C.W., Wang, C.Y.: On the effects of liquid-gas interfacial shear on slip flow through a parallel-plate channel with superhydrophobic grooved walls. Physics of Fluids 22(10), 102,002 (2010)

14. Ng, C.O., Wang, C.Y.: Stokes shear flow over a grating: implications for superhydrophobic slip. Physics of Fluids 21(1), 013,602 (2009)

15. Ng, C.O., Wang, C.Y.: Stokes flow through a periodically grooved tube. Journal of Fluids Engineering 132(10), 101,204 (2010)

16. Ng, C.O., Wang, C.Y.: Effective slip for stokes flow over a surface patterned with two-or three-dimensional protrusions. Fluid Dynamics Research 43(6), 065,504 (2011)

17. Ng, C.O., Zhou, Q.: Electro-osmotic flow through a thin channel with gradually varying wall potential and hydrodynamic slippage. Fluid Dynamics Research 44(5), 055,507 (2012)

18. Nishimoto, S., Bhushan, B.: Bioinspired self-cleaning surfaces with superhydrophobicity, superoleophobicity, and superhydrophilicity. RSC Advances 3(3), 
671-690 (2013)

19. Peskin, C.S.: Numerical analysis of blood flow in the heart. Journal of Computational Physics 25(3), 220-252 (1977)

20. Philip, J.R.: Flows satisfying mixed no-slip and no-shear conditions. Zeitschrift für angewandte Mathematik und Physik ZAMP 23(3), 353-372 (1972)

21. Quéré, D.: Non-sticking drops. Reports on Progress in Physics 68(11), 2495 (2005)

22. Rajagopal, M.C., Das, S.K.: Analyses of drag on viscoelastic liquid infused bioinspired patterned surfaces. Journal of Non-Newtonian Fluid Mechanics 228, 17-30 (2016)

23. Rykaczewski, K., Anand, S., Subramanyam, S.B., Varanasi, K.K.: Mechanism of frost formation on lubricant-impregnated surfaces. Langmuir 29(17), 5230-5238 (2013)

24. Rykaczewski, K., Paxson, A.T., Staymates, M., Walker, M.L., Sun, X., Anand, S., Srinivasan, S., McKinley, G.H., Chinn, J., Scott, J.H.J., et al.: Dropwise condensation of low surface tension fluids on omniphobic surfaces. Scientific Reports 4, 4158 (2014)

25. Schönecker, C., Baier, T., Hardt, S.: Influence of the enclosed fluid on the flow over a microstructured surface in the cassie state. Journal of Fluid Mechanics 740, 168-195 (2014)

26. Schönecker, C., Hardt, S.: Longitudinal and transverse flow over a cavity containing a second immiscible fluid. Journal of Fluid Mechanics 717, 376-394 (2013)

27. Schönecker, C., Hardt, S.: Assessment of drag reduction at slippery, topographically structured surfaces. Microfluidics and Nanofluidics 19(1), 199-207 (2015)

28. Smith, J.D., Dhiman, R., Anand, S., Reza-Garduno, E., Cohen, R.E., McKinley, G.H., Varanasi, K.K.: Droplet mobility on lubricant-impregnated surfaces. Soft Matter 9(6), 1772-1780 (2013)

29. Solomon, B.R., Khalil, K.S., Varanasi, K.K.: Drag reduction using lubricantimpregnated surfaces in viscous laminar flow. Langmuir 30(36), 10,970-10,976 (2014)

30. Subramanyam, S.B., Rykaczewski, K., Varanasi, K.K.: Ice adhesion on lubricant-impregnated textured surfaces. Langmuir 29(44), 13,414-13,418 (2013)

31. Tryggvason, G., Bunner, B., Esmaeeli, A., Juric, D., Al-Rawahi, N., Tauber, W., Han, J., Nas, S., Jan, Y.J.: A front-tracking method for the computations of multiphase flow. Journal of Computational Physics 169(2), 708-759 (2001)

32. Wang, C.Y.: Flow over a surface with parallel grooves. Physics of Fluids 15(5), 1114-1121 (2003)

33. Wexler, J.S., Jacobi, I., Stone, H.A.: Shear-driven failure of liquid-infused surfaces. Physical Review Letters 114(16), 168,301 (2015)

34. Wilson, P.W., Lu, W., Xu, H., Kim, P., Kreder, M.J., Alvarenga, J., Aizenberg, J.: Inhibition of ice nucleation by slippery liquid-infused porous surfaces (SLIPS). Physical Chemistry Chemical Physics 15(2), 581-585 (2013)

35. Wong, T.S., Kang, S.H., Tang, S.K., Smythe, E.J., Hatton, B.D., Grinthal, A., Aizenberg, J.: Bioinspired self-repairing slippery surfaces with pressure-stable 
omniphobicity. Nature 477(7365), 443-447 (2011)

36. Xiao, L., Li, J., Mieszkin, S., Di Fino, A., Clare, A.S., Callow, M.E., Callow, J.A., Grunze, M., Rosenhahn, A., Levkin, P.A.: Slippery liquid-infused porous surfaces showing marine antibiofouling properties. ACS Applied Materials \& Interfaces 5(20), 10,074-10,080 (2013)

37. Yao, X., Hu, Y., Grinthal, A., Wong, T.S., Mahadevan, L., Aizenberg, J.: Adaptive fluid-infused porous films with tunable transparency and wettability. Nature Materials 12(6), 529-534 (2013) 